\title{
A tool to predict perceived urban stress in open public spaces
}

\section{Martin Knöll and Katrin Neuheuser}

Technische Universität Darmstadt, Germany

\section{Thomas Cleff}

Business School Pforzheim at Pforzheim University, Germany

\section{Annette Rudolph-Cleff}

Technische Universität Darmstadt, Germany

\begin{abstract}
This article presents an exploratory framework to predict ratings of subjectively perceived urban stress in open public spaces by analysing properties of the built environment with GIS and Space Syntax. The authors report on the findings of an empirical study in which the environmental properties of a sample of open public spaces in the city of Darmstadt, Germany were constructed and paired to users' ratings. The data are analysed using different types of multivariate analyses with the aim to predict the ratings of perceived urban stress with a high explained variance and significance. The study finds that open public space typologies (park, square, courtyard, streets) are the best predictors for perceived urban stress, followed by isovist characteristics, street network characteristics and building density. Specifically, the isovist visibility, vertices number and perimeter, previously related to arousal and complexity in indoor spaces, show significant relation to perceived urban stress in open public spaces, but with different direction of effects. A model is presented that achieves a predictive power of $R^{2}=54.6 \%$. It extends existing models that focused on green spaces and streetscapes with a first exploratory attempt to predict more complex reactions such as perceived urban stress.
\end{abstract}

\section{Keywords}

Open public space, built environment, perceived urban stress, Space Syntax, GIS

\section{Introduction}

Current city living and upbringing in urban environments have been related to various manifestations of stress and a higher risk for mental health problems. Lederbogen et al. (2013), for instance, have named a set of influencing factors to urban social stress such as

\section{Corresponding author:}

Martin Knöll, Department of Architecture, Technische Universität Darmstadt, El-Lissitzky-Str. I,

D-64287 Darmstadt, Germany.

Email: knoell@stadt.tu-darmstadt.de 
infrastructure, socio-economic factors as well as noise and environmental pollution. Yang and Matthews (2010) explored the associations of social and built environment determinants with self-rated stress, finding that social stress research can be expanded in scope if neighbourhood factors include measures of the built environment. A systematic review from Gong et al. (2016) focussed on the relationship between objective measurements of urban environment and psychological distress. They demand for future work to look at the spatial-temporal dynamic of the urban environment measured in GIS in relation to psychological distress. It remains an open question which aspects of the built environment are critical to influence perceived stress in city dwellers, and how they become effective on different scales of planning. Whereas there is considerable knowledge on how the built environment relates to physical activity (Frank et al., 2003), there is limited research on how environmental properties relate to users' experience while being in motion (Bielik et al., 2015) and to possible psychological impacts (Gong et al., 2016).

Lazarus and Folkman (1984) state that stress is a relationship between the person and the environment that is appraised by the person as taxing or exceeding his or her resources and endangering his or her wellbeing. Assessing stress with a satisfactory objective perspective is difficult. Many existing instruments to measure psychological distress focus on general health outcomes, depression or anxiety for clinical reasons (Eaton et al., 2004; Goldberg and Williams, 1988; Kessler et al., 2002), others are sensitive for examining task stressors (Helton and Näswall, 2015, Müller and Basler, 1993). Following Lazarus (1990), subjective measures like self-rated stress are believed to better capture the combination of environmental demands and coping ability and are an appropriate measure to use in the absence of a complete inventory of stressful events.

With the advancement and greater availability of mobile sensors, researchers have investigated pedestrians' emotions by gaining physiological effects such as heartrate (Song et al., 2013), skin conductance (König et al., 2014) and brainwaves (Aspinall et al., 2013). While these studies offer valuable new insights in the role of green spaces and streetscapes, they are still of explorative nature. All of the above studies use small numbers of test persons and Aspinall et al. (2013) report of limitations in terms of data accuracy. In this article, the authors argue that further challenges result from the fact that there is no taxonomy available that allows describing stress perception of pedestrians using environmental properties. The aim of this article is to investigate which environmental properties are (1) commonly used in urban design research and practice and (2) can be constructed with public available data and (3) do relate or even predict ratings of subjectively perceived stress in a wide spectrum of open public spaces (OPSs) including parks, piazzas, squares and streets.

It addresses the following research questions:

(1) Which properties commonly used to describe the built environment have a significant influence on users' ratings of OPSs as being stressful?

(2) How do they interact with each other and which categories (e.g. typology, isovist and syntactical characteristics and density) have the highest predictive power?

(3) How does a model that can be linked to the built environment through objective measures perform in terms of predictive power compared to other existing models?

The results will be useful for further interdisciplinary research and empirical studies laying the ground to specify relevant properties of the built environment and to identify problematic urban configurations. 


\section{Theoretical background and research hypotheses}

Watts et al. (2013) have presented a tool to predict users' ratings of urban green spaces as being tranquil, which they defined as a quiet, peaceful and restorative place. Their model integrates daytime noise levels from transportation sources, the percentage of natural and contextual features in visual scenery as well as moderating factors. They gained visitors' assessment of a place in face-to-face interviews on the spot in order to predict the most tranquil and the least tranquil area in each of the sample of eight green spaces. Their results show highest correlation between predictions and actual ratings in the most tranquil areas, whereas smaller correlations were found in least tranquil areas. Since the non-tranquil areas are mostly situated on the boundary of green spaces exposed to individual traffic flows, the results are highly dependent on exact assessment positions (Watts et al., 2013). Watts et al.'s (2014) model is shown to predict tranquillity ratings in the sample with a coefficient of determination of $\mathrm{R}^{2}=0.89$ and high significance $(\mathrm{p}<.001)$. It is important to note that Watts et al. (2014) have focused on green spaces only and have used ecological instead of morphologic data including ratings of subjectively perceived tranquillity.

Bielik et al. (2015) investigate how space syntax properties can be used to predict the effect of urban form on a wider scope of environmental appraisal. Using principal component analysis, they reduce 11 adjectives into three main components labelled appeal, activity and spatial experience. Whereas they find the latter two components activity and spatial experience may be well predicted by a single property of urban form, they state that appeal might be more complex and therefore would have to be modelled with multiple urban attributes. They suggest a combination of local attributes such as isovists and of global or relational attributes such as integration to relate to peoples' ratings of appeal. Their model uses properties of built environment only, but was not able to specify aspects of environmental appeal related to urban stress.

Environmental aspects that have been related to various manifestations of urban stress in other studies include noise, crowding of residents, extreme heat waves and air pollution (Evans and Cohen, 1987), motorised traffic (Appleyard and Lintell, 1972) and lack of green space (De Vries, 2010). But so far, there has been no work that investigated perceived stress in a wider range of OPSs.

This article is focussed on those properties that can be directly linked to the physical environment, which Lynch (1981) defined as 'spatial patterns of all large, inert, permanent physical objects in a city such as buildings, streets, utilities, hills, rivers and trees'. The following sections discuss literature, in which properties have been related to stress starting with urban density, isovist characteristics, syntactical properties and typology.

\section{Urban density}

Urban density has been seen influential on city dwellers' mental life and wellbeing since the beginning of modern great cities (Simmel, 2006). More recently, the compact city as a form of distinguishable settlement characterised by a high density of buildings, infrastructure and services, has been related to various positive effects on quality of life and health. Burton (2002) presents a set of commonly used indicators for measuring compactness. She distinguishes high average population density (gross density), high density of built form (net density), a high density of subcentres such as public transport hubs and high streets (decentralised concentration) as well as a high density of compact building forms such as terraced houses and apartments. 
Many studies focus on the relation between the built environment and overall physical activity emphasising density and diversity of use patterns, urban design and street network characteristics (Burton and Mitchell, 2006; Frank et al., 2003). In all those studies, small block sizes are related to less stressful experiences. We included net building density as the variables building coverage ratio, defined as the building footprint per area and floor area ratio, defined as total area of all developed floor space per area (Lee et al., 2011) in our study.

Hypothesis 1: Net building density relates positively to perceived urban stress. Floor area ratio is a better predictor than building coverage ratio, since it covers building heights.

\section{Isovist properties}

In order to assess physical and psychological experiences of outdoor spaces, Osmond (2011) points to the traditional measures such as isovist area, perimeter, maximal radials, occlusivity (Benedikt, 1979) and compactness (Batty, 2001). Isovist area is commonly defined as the amount of space, which is unobstructed by built space of any kind at eye level $(160 \mathrm{~cm})$ seen from a vantage point in an OPS and measured in square meters. Following Osmond (2011), it is assumed that vegetation does not obstruct views since data on users' perception were collected in winter, where the obstruction through leafs is lowest. Visibility is defined as the relative size of isovist areas, retrieved from an analysis of multiple positions by computing the visibility of positions regularly distributed over the whole environment (Turner et al., 2001). Visibility has been positively related to perceived safety in public buildings (Kuliga et al., 2013) and higher pedestrian safety (Stoker et al., 2015).

Hypothesis 2: Isovist area and visibility are negatively associated with perceived urban stress.

Osmond (2011) underlines that isovist area in combination to its perimeter as the total outline are important factors when studying visual stimulation in terms of the information available in a given surrounding environment. Roundness has been defined as the relation between isovist area and its perimeter set in square. It has been related to interestingness as an indicator for arousal and (visual) complexity of an environment in (Franz and Wiener, 2008). Long, thin rectangles (for example, $10 \times 80 \mathrm{~m})$ span a small area $(800 \mathrm{sqm})$ with their perimeter $(180 \mathrm{~m})$, while 'rounder' shapes such as squares (for example, $40 \times 50 \mathrm{~m}$ ) span a bigger area $(2000 \mathrm{sqm})$ with the same perimeter $(180 \mathrm{~m}$ each). High streets are examples for OPSs with big isovist areas, high perimeters and low roundness. In the literature, perimeter and roundness have been related to interestingness and complexity.

Hypothesis 3: Perimeter and roundness are positively associated with perceived urban stress.

From empirical studies with virtual reality environments, Franz and Wiener (2008) also relate openness ratio, vertices number and vertices density to interestingness as an indicator for arousal. Wiener et al. (2007) show that specifically isovist vertices number relates to user ratings of complexity and interestingness. Vertices number is defined as the total amount of vertices of an isovist perimeter and vertices density as standardised index of vertices number divided by isovist total area. A weak relation was found between the vertices density and participants' ratings of safety (Knöll et al., 2015). While interestingness alone may not be a good indicator for perceived stress, we include vertices number, as it has also been related to complexity. Examples for OPSs with high vertices numbers are high streets. 
Hypothesis 4: Vertices number is positively related to perceived urban stress.

Openness ratio is defined as the balance between closed and open edges of an isovist, which has also been considered as being related to interestingness and complexity in indoor spaces (Franz and Wiener, 2008).

Hypothesis 5: Openness ratio is positively associated to perceived urban stress.

\section{Syntactical properties}

The degree of integration of particular street segments in the urban grid has been considered as a pervasive indicator for pedestrian movement patterns (Hillier et al., 1993). It is commonly distinguished between local, citywide and global integration, which indicates how well a street segment is integrated into a wider network of different scales. Wineman et al. (2012) have shown that mean global integration of peoples' home address street segment is positively associated with self-reported physical activities. Sarkar et al. (2013) have used syntactic properties and related them to data of self-reported psychological distress in older men. They found that high global integration $(r=n)$ values, which are collinear with high amounts of motorised traffic, are related with higher odds of psychological distress. In preliminary research, Knöll et al. (2015) could find significant relations between global $(\mathrm{r}=\mathrm{n})$ and citywide $(\mathrm{r}=3000 \mathrm{~m})$ integration values and users' ratings of OPSs as being stressful, relaxing, safe as well as being exposed to traffic. In particular, high global integration values $(\mathrm{r}=\mathrm{n})$ were shown significantly related to ratings of OPSs as stressful. Overall, the analysed literature indicates that line-based syntactical measures such as global and citywide integration may be relevant properties.

Hypothesis 6: Global $(\mathrm{r}=\mathrm{n})$ and citywide $(\mathrm{r}=3000 \mathrm{~m})$ integration are positively related to perceived urban stress.

Sarkar et al. (2013) found that high levels of local-level accessibility, e.g. high values in local integration $(\mathrm{r}=1200 \mathrm{~m})$, are associated with lower odds of psychological distress in older men. According to their explanation, the high value of local integration is associated with high walkability of the area, where respondents would have more access to public life and care facilities. In a preliminary work, Knöll et al. (2015) have found local integration negatively related to ratings of an OPS as being stressful. Axial connectivity is defined as the number of segments that are directly connected to a given street segment. High values in axial connectivity are co-linear with high densities of intersections, suggesting that pedestrians may be more alert to their environment. However, Sarkar et al. (2013) have not found connectivity being significantly related to self-reported psychological distress. As high connectivity is collinear to small block sizes, which have been related to better walkability (Frank et al., 2003) and navigation for people with restricted cognitive and motoric skills (Burton and Mitchell, 2006), we assume connectivity to be negatively related to perceived urban stress.

Hypothesis 7: Local integration $(\mathrm{r}=1200 \mathrm{~m})$ and connectivity are negatively related to perceived urban stress.

\section{Typology}

Much research on health-promoting mechanisms including increased physical activity, social interaction, restoration and stress relief has focused on green spaces 
(Nieuwenhuijsen et al., 2014), leaving a wider spectrum of OPSs including streetscapes and squares to future research. Wolfrum (2014) presents typologies of European squares according to their historical appearance, morphological aspects, geometrical form, size, usages and performative potential. Janson and Bürklin (2002) follow a phenomenological research approach in urban design, in which the perception of the built environment is being assessed by an intuitive combination of theoretical analysis and immediate experience of urban spaces in situ. This approach is helpful for teaching and planning practice. It offers a starting point for this explorative research that seeks to combine the description of OPSs with gaining empirical data on perceived urban stress. In this article, we have included the six categories of square, park, courtyard, heavy traffic street, medium traffic street and pedestrian zone, from which our sample of OPSs was chosen based on names and available traffic data (Knöll et al., 2014).

Hypothesis 8: We assume that parks, courtyards and pedestrian zones are negatively related to perceived urban stress. Heavy traffic streets are positively related to perceived urban stress.

\section{Data collection and sampling}

To investigate the relationship between objective measures and subjective cues on perceived urban stress in OPSs, we use variables from an external dataset of environmental properties as well as variables collected from a self-administered online survey (online Appendix 3) about subjective perceptions related to OPSs, which is introduced in more detail in (Knöll et al., 2014).

The independent variable perceived urban stress is collected from the questionnaire and defined as the user's ratings of an urban environment as being maximum or minimum stressful on a 10-point bipolar scale. The lowest value is ' 1 ' meaning 'not stressful', the highest value is ' 10 ' meaning 'maximum stressful'. It is assumed that high values of perceived urban stress are related to a combination of selected independent variables used to describe the built environment listed in Table 1.

Data of building density were taken from the Masterplan of city of Darmstadt (Germany) in 2015. Analog to Wineman et al. (2012), for each reference point assumed in one OPS, a radius of $250 \mathrm{~m}$ is set to define the lot area for which to calculate density values and ratios.

For constructing the Space Syntax variables, we used Depthmap X software (Varoudis, 2012) with a map of Darmstadt on the basis of OpenStreetMap (OpenStreetMap Foundation, 2015), to which we added footpaths based on satellite photos provided by Google Maps (Google Inc., 2015). The representation of the street network was chosen as a cleaned map of centre line segments, with the radius for integration analysis $(\mathrm{r}=1200 \mathrm{~m}$; $\mathrm{r}=3000 \mathrm{~m} ; \mathrm{r}=\mathrm{n}$ ) chosen according to (Sarkar et al., 2013).

As OPSs, we define outdoor open spaces that can be owned by public or private entities, but are accessible to the general public audience. Knöll et al. (2014) selected the sample of OPSs in Darmstadt within a sector of $500 \mathrm{~m}$ stretching from the industrial Western periphery, across the city center to the Eastern periphery with predominantly low-rise one-family housing. Darmstadt has a population of ca. 150,000 inhabitants and is situated within the Rhine-Main Metropolitan area 30 kilometers south of Frankfurt, Germany. The authors developed a set of 11 bipolar adjectives, which they relate to qualitative data on the perception of OPSs. The scales captured environmental characteristics like safety, vegetation, brightness, noise, maintenance, traffic, spaciousness, liveliness, stressfulness, relaxingness and seating (online Appendix 1). The goal of the research study was to reach respondents with a high expertise in analysing and articulating complex patterns in built environments. Therefore, the authors conducted the empirical research 


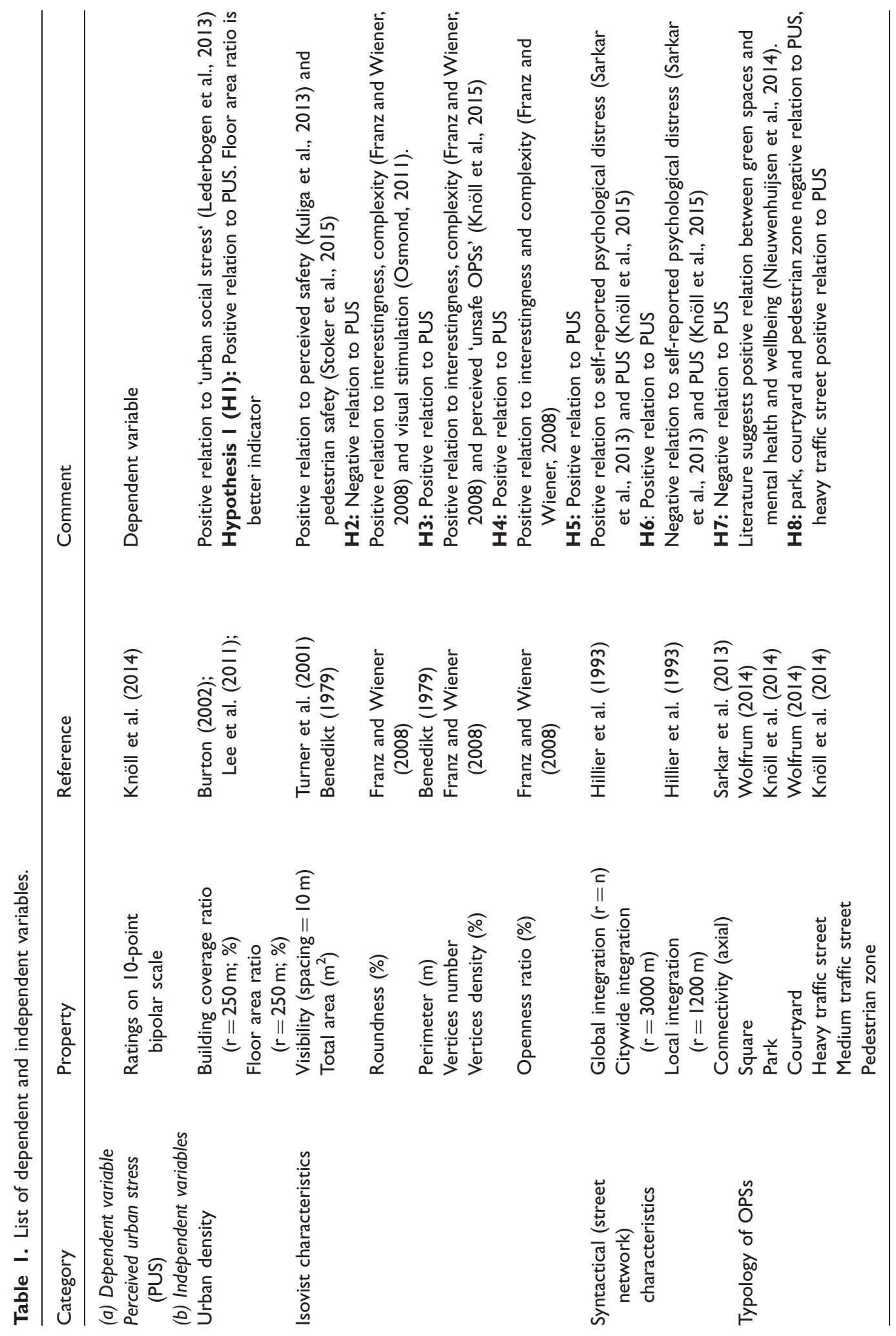


with similarly aged Bachelor students from architecture and urban planning programs at Technische Universität Darmstadt.

A total of 134 respondents completed the survey (online Appendix 3) in December 2013 and in January 2014. Approximately $67 \%$ of respondents were females and $33 \%$ were males, not according to the representative distribution of the German Population. Statistical analyses show that gender has no significant influence on perceived urban stress and therefore has no biasing effects on the results. The same is true for the independent sociodemographic variables age and domicile (see Table 5, Column 1). Around 57\% of the respondents are residents in Darmstadt, the others from neighbouring cities like Frankfurt, Mannheim, Mainz or smaller cities. The age range from 22 to 35 years fulfills range of the sampling frame. The median value and the average age are 25 years. Upper and lower quartiles are 26 and 24 years, respectively, which already indicates small $S D=2.2$.

On the whole, $48.1 \%$ of the respondents rated five OPSs, while $36.3 \%$ rated six OPSs, $14.1 \%$ four OPSs and $1.5 \%$ only one OPS (see Table 2 ).

OPSs within the typology pedestrian zone were rated most often $(22.7 \%)$ followed by heavy traffic streets $(19.2 \%)$ and square $(18.9 \%)$. Courtyards were chosen only for $10.6 \%$ of all ratings (see Table 3 ).

\section{Results}

In the following sections, we will present the single properties in relation to perceived urban stress and the overall performance of the model.

\section{Properties and perceived urban stress}

As anticipated, respondents rate environmental characteristics such as spaciousness, vegetation, brightness, maintenance, traffic, noise, seating, liveliness, safety as very

Table 2. Number of rated open public space (OPS) per respondent.

\begin{tabular}{lcc}
\hline Rated OPS per respondent & Frequency & Valid percent \\
\hline 1 & 2 & 1.5 \\
4 & 19 & 14.1 \\
5 & 65 & 48.1 \\
6 & 49 & 36.3 \\
Total & 135 & 100.0 \\
\hline
\end{tabular}

Table 3. Number of rated open space typologies.

\begin{tabular}{lcc}
\hline Typology & Frequency & Valid percent \\
\hline Courtyard & 74 & 10.6 \\
Park & 93 & 13.3 \\
Medium traffic street & 106 & 15.2 \\
Square & 132 & 18.9 \\
Heavy traffic street & 134 & 19.2 \\
Pedestrian zone & 158 & 22.7 \\
Total & 697 & 100.0 \\
\hline
\end{tabular}


differently for the typologies of OPSs used in this setup (Table 4). The average ratings of an OPS being perceived as 'maximum stressful' lay for all heavy traffic streets at $M=7.52$, for all squares at $M=7.56$, while all parks have been rated as only $M=2.53$ on average.

Around $\mathrm{R}_{\mathrm{R}}^{2}=69.5 \%$ of the variance in perceived urban stress is explained through the variance in ratings of environmental properties. Each variable exerts an influence at least on the five per cent significance level.

In the main analysis, authors included all independent variables described in Table $1-$ which are the urban density, the isovist and street network characteristics and typology - to analyse their influence on perceived urban stress in a multivariate way.

As it can be seen in Table 5 (Column 2), around $\mathrm{R}_{\text {Prop }}^{2}=56.0 \%$ of the variance of the perceived urban stress can be explained only by the defined properties. Adding the dummy variables of the OPSs (see Table 5, Column 4), the coefficient of determination increases about $2.5 \%$ points to $\mathrm{R}_{\text {Prop }}^{2}=58.5 \%$. To improve the coefficient of determination, the variable vertices number is included as squared term and the variables citywide integration, local integration and perimeter as natural logarithmic terms in the model, indicating a curved and non-linear relationship of these variables with perceived urban stress. All variables are highly significant on a $99 \%$ significance level.

Building coverage ratio covers the biggest single share for a property in explaining the variance of perceived urban stress $(17 \%)$. It is followed by the natural logarithmic term of isovist perimeter $(14 \%)$ and the syntactical property of local integration $(13 \%)$, the nonlogarithmic terms square vertices number (12\%) and visibility (11\%) (see Table 6).

The big share and positive relation between building coverage ratio and perceived urban stress is in line with our assumption (Hypothesis 1). Contrary to our assumption, the data show that using floor area ratio decreases the coefficient of determination $\mathrm{R}^{2}$ Prop about $1.2 \%$ points compared to building coverage ratio. This means that building heights in a radius of $250 \mathrm{~m}$ around a vantage point play a relatively small role explaining perceived urban stress in OPSs.

The data show that visibility is positively related to perceived urban stress. This is contrary to our assumption (Hypothesis 2), which we retrieved from literature that has shown visibility positively related to perceived safety in public buildings (Kuliga et al., 2013). The results underline the ambiguity of visibility as the relative size of an area that one can oversee and the area from which one can be seen at a specific point. The data suggest that the latter aspect of visibility - to be seen from a larger area - plays an important role in explaining

Table 4. Mean values for rated typologies.

\begin{tabular}{llllllll}
\hline $\begin{array}{l}\text { Rating } \\
\text { (I-min, I0-max.) }\end{array}$ & Court-yard & $\begin{array}{l}\text { Heavy } \\
\text { traffic street }\end{array}$ & $\begin{array}{l}\text { Medium } \\
\text { traffic street }\end{array}$ & Park & $\begin{array}{l}\text { Pedes } \\
\text { trian Zone }\end{array}$ & Square & Total \\
\hline Spacious & 5.20 & 5.85 & 4.40 & 7.78 & 5.01 & 6.03 & 5.65 \\
Vegetation & 3.66 & 3.49 & 5.37 & 8.09 & 5.07 & 1.95 & 4.47 \\
Brightness & 4.55 & 4.24 & 4.78 & 4.99 & 5.16 & 2.79 & 4.39 \\
Maintenance & 2.74 & 5.56 & 5.29 & 3.51 & 4.47 & 4.01 & 4.40 \\
Traffic & 2.61 & 9.01 & 4.31 & 3.16 & 4.02 & 8.30 & 5.57 \\
Noise & 4.65 & 8.72 & 4.56 & 4.21 & 5.58 & 8.36 & 6.27 \\
Seating & 5.57 & 2.34 & 2.89 & 6.45 & 5.11 & 4.11 & 4.27 \\
Lively & 7.11 & 7.17 & 4.44 & 7.93 & 6.81 & 8.95 & 7.11 \\
Safety & 3.33 & 6.12 & 4.36 & 3.99 & 4.41 & 5.06 & 4.68 \\
Perceived urban stress & 3.30 & 7.52 & 3.86 & 2.53 & 4.73 & 7.56 & 5.22 \\
\hline
\end{tabular}


Table 5. Effects on perceived urban stress.

\begin{tabular}{|c|c|c|c|c|c|}
\hline & & \multicolumn{4}{|c|}{ OLS-regression: dependent variable: perceived urban stress ${ }^{\mathrm{a}, \mathrm{b}}$} \\
\hline & & ( 1 ) & $(2)$ & (3) & $(4)$ \\
\hline \multirow[t]{2}{*}{ (Constant) } & a) & $7.092 * *$ & $-18.622 * *$ & $7.56 I^{* *}$ & -9.112 \\
\hline & & $(1.24 I)$ & $(3.504)$ & $(0.182)$ & $(5.064)$ \\
\hline \multirow{2}{*}{ Ln citywide integration } & a) & & $4.869 * *$ & & 3.856 ** \\
\hline & & & $(0.88 \mathrm{I})$ & & $(1.045)$ \\
\hline \multirow[t]{2}{*}{ Ln local integration } & a) & & $-4.785 * *$ & & $-4.264 * *$ \\
\hline & & & $(0.543)$ & & $(0.604)$ \\
\hline \multirow[t]{2}{*}{ Ln perimeter } & a) & & $1.676 * *$ & & $1.122 * *$ \\
\hline & & & $(0.136)$ & & $(0.206)$ \\
\hline \multirow[t]{2}{*}{ Square vertices number $(1000 *)$} & a) & & $-0.01 * *$ & & $-0.006 * *$ \\
\hline & & & $(0.00 \mathrm{I})$ & & $(0.001)$ \\
\hline \multirow[t]{2}{*}{ Visibility $(1000 *)$} & a) & & $0.860 * *$ & & $0.623^{* *}$ \\
\hline & & & $(0.112)$ & & $(0.154)$ \\
\hline \multirow[t]{2}{*}{ Building coverage ratio } & a) & & $\left.12.02\right|^{* *}$ & & $10.932^{* *}$ \\
\hline & & & $(0.856)$ & & $(1.323)$ \\
\hline \multirow[t]{2}{*}{ Park $(=I)$} & b) & & & $-5.034 * *$ & $-1.036 * *$ \\
\hline & & & & $(0.283)$ & $(0.45 \mathrm{I})$ \\
\hline \multirow[t]{2}{*}{ Heavy traffic street $(=I)$} & b) & & & -0.042 & 0.171 \\
\hline & & & & $(0.257)$ & $(0.334)$ \\
\hline \multirow[t]{2}{*}{ Courtyard $(=1)$} & b) & & & $-4.263 * *$ & $-2.102 * *$ \\
\hline & & & & $(0.304)$ & $(0.483)$ \\
\hline \multirow[t]{2}{*}{ Medium traffic street $(=I)$} & b) & & & $3.703 * *$ & $-0.952^{*}$ \\
\hline & & & & $(0.274)$ & $(0.424)$ \\
\hline \multirow[t]{2}{*}{ Pedestrian zone $(=\mathrm{I})$} & b) & & & $-2.826 * *$ & $-1.228 * *$ \\
\hline & & & & $(0.247)$ & $(0.256)$ \\
\hline \multirow[t]{2}{*}{ Age } & a) & -0.064 & & & \\
\hline & & $(0.049)$ & & & \\
\hline \multirow[t]{2}{*}{ Gender $($ male $=\mathrm{I})$} & b) & -0.154 & & & \\
\hline & & $(0.236)$ & & & \\
\hline \multirow[t]{2}{*}{ Darmstadt $($ Darmstadt $=I)$} & b) & -0.366 & & & \\
\hline & & $(0.220)$ & & & \\
\hline $\mathrm{R}^{2}$ & & 0.008 & 0.560 & 0.463 & 0.585 \\
\hline $\mathrm{F}$ & & 1.839 & $145.998 * *$ & $119.004 * *$ & $87.699 * *$ \\
\hline VIF & & $<1.07$ & $<6.1$ & $<\mathrm{I} .7$ & $<10.4$ \\
\hline Obs. & & 694 & 695 & 695 & 695 \\
\hline
\end{tabular}

a: continuous variable; b: dummy variable. $* *$ and $*$ indicate significance at the $1 \%$ and $5 \%$ levels.

${ }^{\mathrm{a}}$ I0-Point scale.

bMethod: OLS-regression.

perceived urban stress in outdoor spaces. While literature highlights high visibility of a person as key for actual pedestrian safety (Stoker et al., 2015), our results indicate that OPSs with high visibility, for example, a large square with much motorised traffic and 'safe' pedestrian crossings, is still perceived as stressful.

The data confirm that perimeter is positively related to perceived urban stress (Hypothesis 3). Examples for OPSs with high perimeter are heavy traffic streets and squares. The data contradict our assumption that roundness would be a better predictor 
Table 6. Standardised beta coefficients random-effects GLS regression.

\begin{tabular}{|c|c|c|c|}
\hline & \multicolumn{3}{|c|}{ Dependent variable: Urban stress level ${ }^{\mathrm{a}}$} \\
\hline & $\begin{array}{l}\text { Standardised } \\
\text { beta coefficient }\end{array}$ & $\begin{array}{l}\text { Percent of the absolute } \\
\text { sum of all standardised } \\
\text { beta coefficients, } \%\end{array}$ & Sum, \% \\
\hline Building density influence & & & 17.17 \\
\hline Building coverage ratio & $0.514 * *$ & 17.17 & \\
\hline Syntactical influence & & & 20.18 \\
\hline - Ln Citywide Integration & $0.210^{* *}$ & 7.02 & \\
\hline - Ln Local Integration & $-0.394 * *$ & 13.16 & \\
\hline Isovist influence & & & 36.99 \\
\hline - Visibility & $0.321 * *$ & 10.73 & \\
\hline - Ln Perimeter & $0.417^{* *}$ & 13.93 & \\
\hline - Square vertices number & $-0.369 * *$ & 12.33 & \\
\hline Open space typologies influence & & & 25.66 \\
\hline - Park $($ park $=\mathrm{I})$ & $-0.140 *$ & 4.68 & \\
\hline - Heavy traffic streets (heavy traffic $=\mathrm{I}$ ) & 0.100 & 3.34 & \\
\hline - Courtyard (Courtyard =I) & $-0.223 * *$ & 7.45 & \\
\hline - Medium traffic streets (medium traffic $=1$ ) & $-0.124^{*}$ & 4.14 & \\
\hline - Pedestrian zone $($ Pedestrian $=\mathrm{I})$ & $-0.181 * *$ & 6.05 & \\
\hline
\end{tabular}

$* *$ and $*$ indicate significance at the $1 \%$ and $5 \%$ levels.

${ }^{a}$ Random-effects GLS regression [corr $\left(\mathrm{u} \_\mathrm{i}, \mathrm{X}\right)=0$ (assumed)].

for perceived urban stress: Using Roundness instead of perimeter decreases the coefficient of determination $\mathrm{R}_{\text {Prop }}^{2}$ about $1.3 \%$ points.

Contrary to our assumption (Hypothesis 4), the data show that isovist vertices numbers relate negatively to perceived urban stress. While Franz and Wiener (2008) have shown vertices numbers related positively to the perception of complex and visually interesting indoor spaces, vertices numbers are negatively related to perceived urban stress in our sample. The term of square vertices number and the logarithmic term of perimeter effect with alternating directions but similar strength on perceived urban stress. This means that large streetscapes and squares with low vertices numbers, e.g. with low detailing and complexity in the building facades, are more likely to be perceived as stressful. This adds data to architects and planners, who criticise large spaces with less spatially detailed building walls as not in line with human scale (Gehl, 2012).

The data also contradict our assumption (Hypothesis 5) and show that openness ratio is not a significant predictor for perceived urban stress. Using openness ratio instead of perimeter decreases the coefficient of determination $\mathrm{R}^{2}$ Prop again about $1.4 \%$ points. This finding suggests that in outdoor spaces visibility and perimeter, which describe the shape of an OPS and vertices number, which indicates the complexity of a shape, are more important isovist characteristics to explain perceived urban stress.

As assumed in Hypothesis 6, citywide integration is a good indicator to perceived urban stress. This is in line with previous work (Knöll et al., 2015) and may be explained with its strong relation to motorised traffic. Also as assumed (Hypothesis 7), local integration relates negatively to perceived urban stress. Our findings are in line with previous work, in which high local integration, e.g. a high walkability of an area, had been related to lower odds of self-reported psychological distress in older men (Sarkar et al., 2013). This means that a less 
walkable street network also led participants to perceive the surrounding open space as being more stressful.

As assumed in Hypothesis 8, the impact of all six open space typologies accounts for $26 \%$ of explanation of perceived urban stress. In our sample, heavy traffic streets and squares are not statistically different from each other. In comparison, perceived urban stress is rated 1.0 lower in medium traffic streets and parks. It is rated 1.2 lower in pedestrian zones and 2.1 lower in courtyards.

The strong positive correlation between building coverage ratio and perceived urban stress in our sample points to the important role the built environment may play in promoting mental health. However, it is important to note that building coverage ratio is only one ingredient for a model to better understand perceived urban stress. Isovist $(37 \%)$ and syntactical street network $(20 \%)$ characteristics together account for $57 \%$ of the variance of perceived urban stress (Table 6). Citywide integration and local integration act with different directions (different algebraic signs) and strengths on perceived urban stress. As mentioned above, high values in local integration associated with good walkability relate to low values in perceived urban stress, whereas high values in citywide integration associated to motorised traffic are positively related to perceived urban stress. This confirms the importance of measures to reduce and calm motorised traffic in OPSs, giving priority to pedestrians over cars (Stoker et al., 2015). However, the effect of local integration on perceived urban stress is twice as robust as the effect of citywide integration. This may highlight the potential for reducing urban stress with urban design measures focussing on walkability, including smaller block sizes and pedestrian infrastructure.

Conclusively, we have found that visibility and building coverage ratio together explain $28 \%$ of perceived urban stress. This means that high visibility in an area that has also high values in building coverage ratio is positively related to perceived urban stress. In contrast, OPSs with high visibility and low building coverage ratio tend to be perceived as less stressful. The effect of building coverage ratio is 1.5 times stronger than the effect of visibility. The magnitude of the influence of visibility and building coverage ratio can be drawn from the last column of Table 6 . These results underline the call for a wider spectrum of parks and gardens but also 'small public spaces' such courtyards, free lots and squares (Whyte, 1980) providing a greater mix of visibility in OPSs.

\section{Urban stress model}

Having used all properties of the open space typologies as independent dummy variables only, the explained variance for perceived urban stress already reaches a very high value of $\mathrm{R}_{\mathrm{T}}^{2}=46.3 \%$ (Table 5, Column 3). In consequence, it is appropriate to include the properties stemming from the four categories established in Table 1. The coefficient of determination of $\mathrm{R}^{2}{ }_{\mathrm{C}}=58.5 \%$ is being achieved using citywide integration, local integration, perimeter, square of vertices number, visibility, building coverage ratio and typologies (Table 5, Column 4). It may not be as high as $R_{R}^{2}=69.5 \%$ introduced above, which also uses participants' ratings. However, this approach does not require any previous survey questionnaire on users' perception to explain the perceived urban stress. As the observable numbers of rated OPSs per respondent differ between individuals (see Table 2), authors controlled for possible individual fixed and random effects. The results can be drawn from online Appendix 5. Statistical tests ${ }^{1}$ show that there are no biasing fixed effects of the respondents on perceived urban stress. Effects only vary randomly without major changes of regressors compared to the results of the pooled OLS in Column 4 of Table 5 . 
The variance of this equation $\left(\mathrm{R}^{2}=.585\right)$ to predict perceived urban stress is not as high as the variance of the equation presented by Watts et al. (2014) to predict users' assessment of tranquillity in green spaces $\left(\mathrm{R}^{2}=0.89, \mathrm{p}<0.001\right)$. But it is important to note that the model presented here does not require an elaborated and expensive acquisition or analysis of environmental data such as noise levels, or the subjective assessment of users of maintenance conditions. The model presented here achieves a reasonable predictive power with data gained merely from an analysis of the built environment. All properties can be derived from tools such as GIS or Space Syntax.

\section{Conclusions}

This article presents a method to predict ratings of perceived urban stress in outdoor spaces. It is a first attempt to predict more complex emotions such as perceived stress with a set of environmental properties that can be derived from commonly used spatial analysis tools such as GIS and Space Syntax. It further develops the current approaches, which have focussed on appeal and have not aimed for explaining more complex emotions such as stress (Bielik et al., 2015). The model presented here is a first approach in this direction to analyse perceived urban stress. It may be particularly useful to identify problematic locations in a preliminary analysis for further and more detailed investigations.

A regression model is presented that uses a combination of environmental properties to predict perceived urban stress and achieves a predictive power of $\mathrm{R}^{2}=58.5 \%$. The results further specify recent findings, which have related living in urban environments to higher levels of urban social stress with four categories of environmental properties to describe perceived urban stress: (a) open space typology, (b) syntactical and (c) isovist characteristics and (d) urban density. The study finds that open space typologies (park, square, courtyard, streets) are the best predictors for perceived urban stress. They are followed by single properties such as building coverage ratio, isovist vertices numbers, local integration and citywide integration. Our data show that the literature relating isovist characteristics and human perception in indoor spaces can only be extended to outdoor spaces to a limited degree. In fact, perimeter was the only variable, which was confirmed as a good predictor with a similar direction of effects when used in indoor and outdoor spaces. Visibility and vertice number relate to urban perceived stress with changed directions, while openness ratio was not found to be relevant at all. This is contrary to our assumptions, and in contrast to the results previously reported on virtual indoor spaces (Franz and Wiener, 2008) and in public buildings (Kuliga et al., 2013). Overall, our study specifies a first set of environmental characteristics related to perceived urban stress in outdoor spaces. However, our findings call for more empiric studies on isovist characters particularly for outdoor spaces.

Specifically, it remains an open question how the variables presented in this article, interact with each other. The presented data show that high building coverage ratio is positively related to perceived urban stress, while high isovist vertices numbers (indicating complex buildings walls of OPSs) are related negatively to perceived urban stress. We found that high local integration of street segments (indicating high volumes of pedestrian movement) relates negatively to perceived urban stress, while high citywide and global integration (indicating higher volumes of car traffic) relate positively to perceived urban stress.

The article explores the role of perceived urban stress in creating quality open spaces that invite physical activity and promote mental health. The results highlight that next to mostly static variables such as OPS typology and building density, more dynamic variables such as local integration of street segments as well as isovist characteristics play a key role in 
perceived urban stress. These latter dynamic variables can be influenced and should be addressed for instance by traffic calming or by urban design measures. Future research should address the interaction of variables in more depth. More empiric data would allow planners and decision makers to prioritise and orchestrate design interventions with respect to mental health and stress. The framework presented here may be used to describe the built environment in more detail in further interdisciplinary research.

\section{Strengths and limitations}

The sampling of test persons is been helpful to get a good response in terms of assessing environmental qualities. In fact, the authors choose students of architecture and urban planning as participants in this first explorative study, since they are particularly sensitive towards the built environment. However, they are not representative for a wider population as they do represent a certain age group, educational and cultural background and occupation. Future research should expand this study to a wider spectrum of the population.

The sampling of the OPSs in Darmstadt includes a wide spectrum of typologies, geometric shapes, locations and context. The resulting model is robust in so far as it can be generalised to other OPSs in Darmstadt or other similar other cities. Since the properties used can be retrieved or constructed from available data, the model can be used to visualise perceived urban stress, for instance as part of a heat map on urban stress for Darmstadt. However, it remains open how the results can be generalised to other types of cities. Darmstadt is only representative for a middle-sized city in a central European context. Future studies must confirm and extend findings in settlements with lower and bigger population, first in a central European context with similar cultural and climatic backgrounds and eventually in a global perspective.

Stress perception was only assessed with an online questionnaire, in which test persons have to assess OPSs from their memory. This approach was chosen to maximise the sample of OPSs rated by participants. Future research should also include assessing OPSs on site, for example using location-based applications and bio signals.

Also the construction of environmental properties, especially the isovist characteristics can be improved. Future studies should consider polygon-based measures of isovist analysis, which has been used in indoor spaces (Kuliga et al., 2013). The used open space typologies can also be further developed in future research. It may be interesting if current categories available from geo maps such as open street maps and Google may predict perceived urban stress.

\section{Acknowledgements}

The authors are grateful to Mr. Yang Li, Doctoral Candidate at TU Darmstadt, Department of Architecture, who contributed to the data analysis with Space Syntax and GIS.

\section{Declaration of conflicting interests}

The author(s) declared no potential conflicts of interest with respect to the research, authorship, and/or publication of this article.

\section{Funding}

The author(s) received no financial support for the research, authorship, and/or publication of this article 


\section{Note}

1. No significance of the Hausman test (1978): chi $^{2}(9)=(b-B)^{\prime}\left[\left(V_{-} b-V \_B\right)(-1)\right](b-B)=5.74$ (Prob $>\operatorname{chi}^{2}=0.7656$ ); Significance of the Breusch and Pagan (1980) Lagrangian multiplier test for random effects: chibar2 $(01)=12.10($ Prob $>$ chibar2 $=0.0003)$.

\section{References}

Appleyard D and Lintell M (1972) The environmental quality of city streets: The residents' viewpoint. Journal of the American Institute of Planners 38: 84-101.

Aspinall P, Mavros P, Coyne R, et al. (2013) The urban brain: Analysing outdoor physical activity with mobile EEG. British Journal of Sports Medicine 49(4): 272-276.

Batty M (2001) Exploring isovist fields: Space and shape in architectural and urban morphology. Environment and Planning B: Planning and Design 28: 123-150.

Benedikt ML (1979) To take hold of space: Isovists and isovist fields. Environment and Planning B: Planning and Design 6: 47-65.

Bielik M, Schneider S, Kuliga S, et al. (2015) Investigating the effect of urban form on the environmental appraisal of streetscapes. In: Karimi K, Vaughan L, Sailer K, et al. (eds) Proceedings of the 10th international Space Syntax symposium. London, UK, 13-17 July 2015, pp.119.1-119.13. London: Space Syntax Laboratory.

Breusch TS and Pagan AR (1980) The Lagrange multiplier test and its applications to model specification in econometrics. Review of Economic Studies 47: 239-253.

Burton E (2002) Measuring urban compactness in UK towns and cities. Environment and Planning B: Planning and Design 29: 219-250.

Burton E and Mitchell L (2006) Inclusive Urban Design: Streets for Life. Oxford: Architectural Press.

De Vries S (2010) Nearby nature and human health: Looking at mechanisms and their implications. In: Thompson CW, Aspinall P and Bell S (eds) Innovative Approaches to Researching Landscapes and Health. London: Routledge, pp. 97-99.

Eaton WW, Muntaner C, Smith C, et al. (2004) Center for Epidemiologic Studies Depression Scale: Review and revision (CESD and CESD-R). In: Maruish ME (ed.) The Use of Psychological Testing for Treatment Planning and Outcomes Assessment. Mahwah, NJ, London: Lawrence Erlbaum Associates, pp. 363-377.

Evans WG and Cohen S (1987) Environmental stress. In: Stokols D and Altman I (eds) Handbook of Environmental Psychology. New York: John Wiley \& Sons, pp. 571-610.

Frank LD, Engelke PO and Schmid TL (2003) Health and Community Design: The Impact of the Built Environment on Physical Activity. Washington, DC: Island Press.

Franz G and Wiener JM (2008) From space syntax to space semantics: A behaviourally and perceptually oriented methodology for the efficient description of the geometry and the topology of environments. Environment and Planning B: Planning and Design 15: 574-592.

Gehl J (2012) Cities for People. London: Island Press.

Goldberg D and Williams P (1988) A user's guide to the General Health Questionnaire. Windsor, Berks: NFER-Nelson.

Gong Y, Palmer S, Gallacher J, et al. (2016) A systematic review of the relationship between objective measurements of the urban environment and psychological distress. Environment International 96: $48-57$.

Google Inc. (2015) Google Maps. Available at: https://www.google.de/maps/@49.8653349,8.6470592, $10028 \mathrm{~m} /$ data $=! 3 \mathrm{~m} 1 ! 1 \mathrm{e} 3$ (accessed 6 February 2015).

Hausman JA (1978) Specification tests in econometrics. Econometrica 46: 1251-1271.

Helton WS and Näswall K (2015) Short Stress State Questionnaire: Factor structure and state change assessment. European Journal of Psychological Assessment 31: 20-30.

Hillier B, Penn A, Hanson J, et al. (1993) Natural movement: Or, configuration and attraction in urban pedestrian movement. Enviornment and Planning B: Planning and Design 10: 29-66.

Janson A and Bürklin T (2002) Auftritte/Scenes: Studies of Architectural Space - The Campi of Venice. Zürich: Birkhäuser. 
Kessler RC, Andrews G, Colpe LJ, et al. (2002) Short screening scales to monitor population prevalences and trends in non-specific psychological distress. Psychological Medicine 32(6): 959-976.

Knöll M, Neuheuser K, Li Y, et al. (2015) Using space syntax to analyse stress perception in open public space. In: Karimi K, Vaughan L, Sailer K, et al. (eds) Proceedings of the 10th international Space Syntax symposium. London UK, 13-17 July 2015, pp.1-13: London: Space Syntax Laboratory.

Knöll M, Neuheuser K, Vogt, et al. (2014) Einflussfaktoren der gebauten Umwelt auf wahrgenommene Aufenthaltsqualität bei der Nutzung städtischer Räume. Umweltpsychologie 18(2): 84-102.

König R, Schneider S, Hamzi I, et al. (2014) Using geo statistical analysis to detect similarities in emotional responses of urban walkers to urban space. In: Proceedings of design, computing \& cognition 2014 (DCC14), London, UK, 23-25 June 2014, pp.41-43. London: University College London.

Kuliga S, Dalton-Conroy R and Hölscher C (2013) Aesthetic and emotional appraisal of the seattle public library and its relation to spatial configuration. In: Kim YO, Park HT and Seo KW (eds) Proceedings of the Ninth international Space Syntax symposium. Seoul, 31 October-3 November 2013, pp.77.1-77.17. Seoul: Sejong University Press.

Lazarus RS (1990) Theory-based stress measurement. Psychological Inquiry 1(1): 3-13.

Lazarus RS and Folkman S (1984) Stress, Appraisal, and Coping. New York: Springer.

Lederbogen F, Haddad L and Meyer-Lindenberg A (2013) Urban social stress - Risk factor for mental disorders. The case of schizophrenia. Environmental Pollution 183: 2-6.

Lee T, Imai R, Duffy C, et al. (2011) The density atlas. (Massachusetts Institute of Technology). Available at: http://densityatlas.org/measuring/metrics.shtml (accessed 21 December 2015).

Lynch K (1981) Good City Form. Cambridge, MA: MIT Press.

Müller B and Basler HD (1993) Kurzfragebogen zur Aktuellen BEANSPRUCHUNG (KAB). Göttingen: Hogrefe.

Nieuwenhuijsen MJ, Kruize H, Gidlow C, et al. (2014) Positive health effects of the natural outdoor environment in typical populations in different regions in Europe (PHENOTYPE): A study programme protocol. BMJ Open 4(4): 1-11.

OpenStreetMap Foundation (2015) OpenStreetMap. Avaialble at: http://www.openstreetmap.org/ \#map =14/49.8730/8.6353 (accessed 10 June 2015).

Osmond P (2011) The convex space as the 'Atom' of urban analysis. The Journal of Space Syntax 2(1): 97-114.

Sarkar C, Gallacher J and Webster C (2013) Urban built environment configuration and psychologcial distress in older men: Results from Caerhilly study. BMC Public Health 13(695): 1-12.

Simmel G (2006) Die Großstadt und das Geistesleben. Frankfurt: Suhrkamp.

Song C, Joung D, Ikei H, et al. (2013) Physiological and psychological effects of walking on young males in urban parks in winter. Journal of Physiological Anthroplogy 32: 18.

Stoker P, Garfinkel-Castro A, Khayesi M, et al. (2015) Pedestrian safety and the built environment: A review of the risk factors. Journal of Planning Literature 30(4): 377-392.

Turner A, Doxa M, O'Sullivan D, et al. (2001) From isovists to visibility graphs: a methodology for the analysis of architectural space. Environment and Planning B: Planning and Design 28(1): 103-121.

Varoudis T (2012) DepthmapX Multi-Platform Spatial Network Analysis Software, Version 0.30 Open Source. Avaialble at: http://varoudis.github.io/depthmapX (accessed 12 June 2015).

Watts G, Miah A and Pheasant R (2013) Tranquility and soundscapes in urban green spaces Predicted and actual assessments from a questionnaire survey. Environment and Planning B: Planning and Design 40(1): 170-181.

Watts G, Pheasant R and Horoshenko K (2014) Predicting perceived tranquillity in urban parks and open spaces. Environment and Planning B: Planning and Design 38(4): 585-594.

Whyte WH (1980) The Social Life of Small Public Spaces. New York: Project for Public Spaces.

Wiener JM, Franz G, Rossmanith N, et al. (2007) Isovist analysis captures properties of space relevant for locomotive and experience. Perception 37: 1066-1083. 
Wineman JD, Marans RW, Schulz AJ, et al. (2012) Neighborhood design and health: Characteristics of the built environment and health-related outcomes for residents of Detroit neighborhoods. In: Greene M, Reyes J and Castro A (eds) Proceedings of the 8th International Space Syntax Symposium. Santiago, de Chile: PUC. 3-6 January 2012, pp. 8236.1-8236.12.

Wolfrum S (2014) Squares: Urban Spaces in Europe. Zurich: Birkhäuser.

Yang TC and Matthews SA (2010) The role of social and built environments in predicting self-rated stress: A multilevel analysis in Philadelphia. Health and Place 16(5): 803-810.

Martin Knöll is Junior Professor and head of the Urban Health Games research group in the Department of Architecture at Technische Universität Darmstadt. He is a registered architect and holds a PhD from Universität Stuttgart, Germany, on health-oriented urbanism. Dr. Knöll leads the research group in building new transdisciplinary collaborations between designers and health experts to address global health challenges such as social inclusion, physically inactive lifestyles, diabetes and stress. Previously, Dr. Knöll was visiting scholar at the Lansdown Centre for Electronic Arts at Middlesex University, London, UK, and held a post-doc position in the Department of Architecture at Karlsruhe Institute of Technology, Germany.

Katrin Neuheuser is Research Associate and $\mathrm{PhD}$ student at Technische Universität Darmstadt, Department of Psychology. She has several years' professional experience as physiotherapist and earned her Master's degree in psychology from TU Darmstadt. As part of the Urban Health Games Unit at TU Darmstadt, she is investigating how urban design aspects influence (Knöll, Neuheuser, Cleff, \& Rudolph-Cleff, 2016) human health and wellbeing. Further research interests concern physiological responses to stress, e.g. heart rate variability and cortisol, immunoglobulin A, Oxytocin in saliva.

Thomas Cleff is Professor of Quantitative Methods for Business and Economics at Pforzheim University, Germany. He holds degrees in Economics and in Management Sciences from Pantheon-Sorbonne University, France, and the University of Wuppertal, Germany, where he also earned his doctorate. His main research interests are international marketing research, white-collar crime, brand research, innovation research and industry studies. Part of his research is carried out at the Centre for European Economic Research (ZEW) in Mannheim, where he is a research associate.

Annette Rudolph-Cleff is Professor and head of the chair of urban design and development at Technische Universität Darmstadt, Department of Architecture. She is a registered architect and holds a PhD from the University of Karlsruhe, Germany, on housing policy and urban development. Dr. Rudolph-Cleff is involved in international research projects on urbanisation and locality, semi-centralised supply and treatment systems for fast-growing urban areas. Annette has received many prestigious awards for her work on sustainable urban development, of which one of the latest was the Greentech Award in the category 'Urbanization' (2015). 